JouRnal of Universal Mathematics

Vol.1 No.2 PP.116-129 (2018)

ISSN-2618-5660

\title{
CONSTRUCTION OF A TOPOLOGICAL DEGREE THEORY IN GENERALIZED SOBOLEV SPACES
}

\author{
M. AIT HAMMOU AND E. AZROUL
}

\begin{abstract}
In this paper, we construct an integer-valued degree function in a suitable classes of mappings of monotone type, using a complementary system formed of Generalized Sobolev Spaces in which the variable exponent $p \in \mathcal{P}^{\log }(\Omega)$ satisfy $1<p^{\prime-} \leq p^{+} \leq \infty$, where $\Omega \subset \mathbb{R}^{N}$ is open and bounded. This kind of spaces are not reflexives.
\end{abstract}

\section{INTRODUCTION}

Topological degree theory is one of the most effective tools in solving nonlinear equations.

Brouwer had published a degree theory in 1912 for continuous maps defined in finite dimensional Euclidean space [4]. Leray and Schauder developed the degree theory for compact operators in infinite dimensional Banach spaces [12]. Since then numerous generalizations and applications have been investigated in various ways of approach (see e.g. $[7,15,16,18]$ ). Browder introduced a topological degree for nonlinear operators of monotone type in reflexive Banach spaces $[5,6]$. The theory was constructed later by Berkovits and Mustonen by using the Leray-Schauder degree $[1,2,3]$ which can be applied to partial differential operators of general divergence form.

The purpose of this article is to generalize this theory to Sobolev spaces with variable exponent in the case where these spaces are not reflexives, exactly in the case where the variable exponent $\mathrm{p}$ satisfies $1<p^{{ }^{-}} \leq{p^{\prime}}^{+} \leq \infty$ where $p^{\prime}$ is the dual variable exponent of $p$. We will construct this theory for appropriate classes of monotone mappings using a complementary system formed of Generalized Sobolev spaces.

The paper is divided into three parts. In the second section, we introduce some preliminary definitions and results concerning the generalized Lebesgue and Sobolev spaces, we construct a complementary system of these spaces and we present some classes of monotone cartography. The third section is dedicated to the construction of degree theory in generalized Sobolev spaces.

Date: July 9, 2018, accepted.

2000 Mathematics Subject Classification. Primary Topological degree, Generalized Sobolev spaces; Secondary Complementary system.

Key words and phrases. 47H11, 46E35. 


\section{Preliminary Definitions AND Results}

In the sequel, we consider a naturel number $N \geq 1$ and an open and bounded domain $\Omega \subset \mathbb{R}^{N}$ with segment property.

2.1. Generalized Lebesgue spaces. We define $\mathcal{P}(\Omega)$ to be the set of all measurable function: $p: \Omega \rightarrow[1,+\infty]$. Functions $p \in \mathcal{P}(\Omega)$ are called variable exponents on $\Omega$. We define $p^{-}=\operatorname{ess} \inf _{\Omega} p$ and $p^{+}=\operatorname{ess} \sup _{\Omega} p$.

If $p \in \mathcal{P}(\Omega)$, then we define $p^{\prime} \in \mathcal{P}(\Omega)$ by $\frac{1}{p(x)}+\frac{1}{p^{\prime}(x)}=1$, where $\frac{1}{\infty}:=0$. The function $p^{\prime}$ is called the dual variable exponent of $p$.

We say that a function $\alpha: \Omega \rightarrow \mathbb{R}$ is locally log-Hölder continuous on $\Omega$ if there exists $c_{1}>0$ such that

$$
|\alpha(x)-\alpha(y)| \leq \frac{c_{1}}{\log (e+1 /|x-y|)}
$$

for all $x, y \in \Omega$. We say that $\alpha$ satisfies the log-Hölder decay condition if there exist $\alpha_{\infty} \in \mathbb{R}$ and a constant $c_{2}>0$ such that

$$
\left|\alpha(x)-\alpha_{\infty}\right| \leq \frac{c_{2}}{\log (e+|x|)}
$$

for all $x \in \Omega$. We say that $\alpha$ is globally log-Hölder continuous in $\Omega$ if it is locally log-Hölder continuous and satisfies the log-Hölder decay condition.

We define the following class of variable exponents

$$
\mathcal{P}^{\log }(\Omega):=\left\{p \in \mathcal{P}(\Omega): \frac{1}{p} \text { is globally log-Hölder continuous }\right\} .
$$

We can deduce that $p \in \mathcal{P}(\Omega)$ if and only if $p^{\prime} \in \mathcal{P}(\Omega)$.

For $t \geq 0, x \in \Omega$ and $1 \leq p<\infty$ we define

$$
\varphi_{p(x)}(t):=t^{p(x)}
$$

Moreover we set

$$
\varphi_{\infty}(t):=\infty \cdot \chi_{(1, \infty)}(t)=\left\{\begin{array}{ll}
0 & \text { if } t \in[0,1] \\
\infty & \text { if } t \in(1, \infty)
\end{array} .\right.
$$

We will use $t^{p(x)}$ as an abbreviation for $\varphi_{p(x)}(t)$, also in the case $p=\infty$. Similarly, $t^{\frac{1}{p(x)}}$ will denote the inverse function $\varphi_{p(x)}^{-1}(t)$; note that in case $p=\infty$ we have $t^{\frac{1}{\infty}}=\varphi_{\infty}^{-1}(t)=\chi_{(0, \infty)}(t)$.

For any variable exponent $p(\cdot)$ and any measurable function $u$, we define the modular

$$
\rho_{p(\cdot)}(u)=\int_{\Omega}|u(x)|^{p(x)} d x,
$$

and we define the variable exponent Lebesgue space

$$
L^{p(\cdot)}(\Omega)=\left\{u ; u: \Omega \rightarrow \mathbb{R} \text { is measurable and } \rho_{p(\cdot)}(\lambda u)<\infty \text { for some } \lambda>0\right\}
$$

equipped with the norm, called the Luxemburg norm,

$$
\|u\|_{p(\cdot)}=\inf \left\{\lambda>0 / \rho_{p(\cdot)}\left(\frac{u}{\lambda}\right) \leq 1\right\} .
$$

It is a Banach space $\left(\left[8\right.\right.$, Theorem 3.2.7]). The space $E^{p(\cdot)}(\Omega)$ is the closure of the space $L^{\infty}(\Omega)$ with respect to the Luxemburg norm. 
Theorem 2.1. [8] Let $p(\cdot)$ and $q(\cdot)$ be the exponent and $\Omega \subset \mathbb{R}^{N}$ open and bounded. Then

(i): $E^{p(\cdot)}(\Omega) \subset L^{p(\cdot)}(\Omega)$,

(ii): $E^{p(\cdot)}(\Omega)=L^{p(\cdot)}(\Omega)$ iff $p^{+}<\infty$,

(iii): $E^{p(\cdot)}(\Omega)$ is separable,

(iv): $\left(E^{p(\cdot)}(\Omega)\right)^{*}=L^{p^{\prime}(\cdot)}(\Omega)$,

$(\mathbf{v})$ : $L^{p(\cdot)}(\Omega)$ is reflexive iff $1<p^{-} \leq p(x) \leq p^{+}<\infty$.

We say that a sequence $\left\{u_{n}\right\} \subset L^{p(\cdot)}(\Omega)$ converges to $u \in L^{p(\cdot)}(\Omega)$ in the modular sense, denote $u_{n} \rightarrow u(\bmod )$ in $L^{p(\cdot)}$, if there exists $\lambda>0$ such that

$$
\rho_{p(\cdot)}\left(\frac{u_{n}-u}{\lambda}\right) \rightarrow 0, \text { when } n \rightarrow \infty .
$$

Let $X$ and $Y$ be arbitrary Banach spaces with bilinear bicontinuous pairing $\langle., .\rangle_{X, Y}$. We say that a sequence $u_{n} \subset X$ converges to $u \in X$ with respect the topology $\sigma(X, Y)$, denote $u_{n} \rightarrow u(\sigma(X, Y))$ in $X$, if $\left\langle u_{n}, v\right\rangle \rightarrow\langle u, v\rangle$ for all $v \in Y$. When $Y^{*} \cong X$, we denote only $u_{n} \rightarrow u$ in $X$.

Theorem 2.2. ([8,11]) In any Generalized Lebesgue space $L^{p(\cdot)}(\Omega)$

(i): norm convergence implies modular convergence,

(ii): norm convergence and modular convergence are equivalent iff $p^{+}<\infty$,

(iii): modular convergence implies $\sigma\left(L^{p(\cdot)}, L^{p^{\prime}(\cdot)}\right)$ convergence.

\subsection{Generalized Sobolev spaces and complementary system.}

Definition 2.3. Let $Y$ and $Z$ be Banach spaces in duality with respect with to a continuous pairing $\langle.,$.$\rangle and let Y_{0}$ and $Z_{0}$ be closed subspaces of $Y$ and $Z$ respectively. Then the quadruple $\left(\begin{array}{cc}Y & Z \\ Y_{0} & Z_{0}\end{array}\right)$ is caled a complementary system if, by means of $\langle.,\rangle,. Y_{0}^{*} \cong Z$ and $Z_{0}^{*} \cong Y$.

An example of a complementary system is

$$
\left(\begin{array}{cc}
L^{p(\cdot)}(\Omega) & L^{p^{\prime}(\cdot)}(\Omega) \\
E^{p(\cdot)}(\Omega) & E^{p^{\prime}(\cdot)}(\Omega)
\end{array}\right)
$$

The following lemma gives an important method by which from a complementary system $\left(\begin{array}{cc}Y & Z \\ Y_{0} & Z_{0}\end{array}\right)$ and a closed subspace $E$ of $Y$, one can construct a new complementary system $\left(\begin{array}{cc}E & F \\ E_{0} & F_{0}\end{array}\right)$. Define $E_{0}=E \cap Y_{0}, F=Z / E_{0}^{\perp}$ and $F_{0}=\left\{z+E_{0}^{\perp} ; z \in Z_{0}\right\} \subset F$, where $\perp$ denotes the orthogonal in the duality $(Y, Z)$, i.e. $E_{0}^{\perp}=\left\{z \in Z ;\langle y, z\rangle=0\right.$ for all $\left.y \in E_{0}\right\}$.

Lemma 2.4. [10, Lemma 1.2] The pairing $\langle.,$.$\rangle between Y$ and $Z$ induces a pairing between $E$ and $F$ if and only if $E_{0}$ is $\sigma(Y, Z)$ dense in $E$. In this case, $\left(\begin{array}{cc}E & F \\ E_{0} & F_{0}\end{array}\right)$ is a complementary system if $E$ is $\sigma\left(Y, Z_{0}\right)$ closed, and conversely, when $Z_{0}$ is complete, $E$ is $\sigma\left(Y, Z_{0}\right)$ closed if $\left(\begin{array}{cc}E & F \\ E_{0} & F_{0}\end{array}\right)$ is a complementary system.

Next, let $p \in \mathcal{P}(\Omega)$ and $m \in \mathbb{N}$.

We define the spaces

$$
W^{m, p(.)}(\Omega)=\left\{u \in L^{p(.)}(\Omega): D^{\alpha} u \in L^{p(.)}(\Omega),|\alpha| \leq m\right\},
$$




$$
H^{m, p(.)}(\Omega)=\left\{u \in E^{p(.)}(\Omega): D^{\alpha} u \in E^{p(.)}(\Omega),|\alpha| \leq m\right\}
$$

with the norm

$$
\|u\|_{m, p(.)}=\sum_{|\alpha| \leq m}\left\|D^{\alpha} u\right\|_{p(.)} .
$$

The spaces $W^{m, p(.)}(\Omega)$ and $H^{m, p(.)}(\Omega)$ are Banach spaces (see [8]).

We say that a sequence $\left\{u_{n}\right\} \subset W^{m, p(.)}(\Omega)$ converges to $u \in W^{m, p(.)}(\Omega)$ in the modular sense, denote $u_{n} \rightarrow u(\bmod )$ in $W^{m, p(.)}$, if there exists $\lambda>0$ such that

$$
\rho_{p(.)}\left(\frac{D^{\alpha} u_{n}-D^{\alpha} u}{\lambda}\right) \rightarrow 0, \text { when } n \rightarrow \infty,
$$

for $|\alpha| \leq m$.

The Sobolev space $W_{0}^{m, p(.)}(\Omega)$ with zero boundary valus is the closure of the set of $W^{m, p(.)}(\Omega)$-functions with compact support, i.e.

$$
\left\{u \in W^{m, p(.)}(\Omega): u=u \chi_{K} \text { for a compact } K \subset \Omega\right\}
$$

in $W^{m, p(.)}(\Omega)$.

The space $W^{m, p(.)}(\Omega)$ will always be identified to a subspace of the product $\Pi_{|\alpha| \leq m} L^{p(.)}=\Pi L^{p(.)} ;$ this subspace is $\sigma\left(\Pi L^{p(.)}, \Pi E^{p^{\prime}(.)}\right)$ closed and $W_{0}^{m, p(.)}(\Omega)$ will be the $\sigma\left(\Pi L^{p(.)}, \Pi E^{p^{\prime}(.)}\right)$ closure of $\mathcal{D}(\Omega)=\bigcap_{m=1}^{\infty} C_{0}^{m}(\Omega)$ in $W^{m, p(.)}(\Omega)$.

The (norm) closure of $\mathcal{D}(\Omega)$ in the space $W^{m, p(.)}(\Omega)$ (or in $\left.\Pi L^{p(.)}\right)$ is denoted by $H_{0}^{m, p(.)}(\Omega)$.

If $p \in \mathcal{P}^{\log }(\Omega)$ is bounded, then $W_{0}^{m, p(.)}(\Omega)=H_{0}^{m, p(.)}(\Omega)$ [8, Corollary 11.2.4]. The space $W_{0}^{m, p(.)}(\Omega)$ is a Banach space, which is separable if $p$ is bounded, and reflexive and uniformly convex if $1<p^{-} \leq p^{+}<\infty$ [8, Theorem 8.1.13].

Let $p \in \mathcal{P}^{\log }(\Omega)$ satisfy $1<p^{\prime-} \leq{p^{\prime}}^{+} \leq \infty$. We denote the dual spaces of Sobolev spaces $W_{0}^{m, p(.)}(\Omega)$ and $H_{0}^{m, p(.)}(\Omega)$ as follows

$$
W^{-m, p^{\prime}(.)}(\Omega):=\left(W_{0}^{m, p(\cdot)}(\Omega)\right)^{*} \text { and } H^{-m, p^{\prime}(.)}(\Omega):=\left(H_{0}^{m, p(.)}(\Omega)\right)^{*} .
$$

Proposition 1. [8, Proposition 12.3.2] Let $\Omega \subset \mathbb{R}^{N}$ be a domain, let $p \in \mathcal{P}^{\log }(\Omega)$ satisfy $1<p^{\prime-} \leq p^{\prime+} \leq \infty$ and let $m \in \mathbb{N}$. For each $F \in W^{-m, p^{\prime}(.)}(\Omega)$ there exists $f_{\alpha} \in L^{p^{\prime}(.)}(\Omega),|\alpha| \leq m$, such that

$$
\langle F, u\rangle=\sum_{|\alpha| \leq m} \int_{\Omega} f_{\alpha} D^{\alpha} u d x
$$

for all $W_{0}^{m, p(.)}(\Omega)$. Moreover,

$$
\|F\|_{-m, p^{\prime}(\cdot)} \approx \sum_{|\alpha| \leq m}\left\|f_{\alpha}\right\|_{p^{\prime}(\cdot)} .
$$

We can write an analogue proposition for $H^{-m, p^{\prime}(.)}(\Omega)$ and then

$$
\begin{aligned}
& W^{-m, p^{\prime}(.)}(\Omega)=\left\{F \in \mathcal{D}^{\prime}(\Omega): F=\sum_{|\alpha| \leq m}(-1)^{|\alpha|} D^{\alpha} f_{\alpha}, \text { where } f_{\alpha} \in L^{p^{\prime}(.)}(\Omega)\right\}, \\
& H^{-m, p^{\prime}(.)}(\Omega)=\left\{F \in \mathcal{D}^{\prime}(\Omega): F=\sum_{|\alpha| \leq m}(-1)^{|\alpha|} D^{\alpha} f_{\alpha}, \text { where } f_{\alpha} \in E^{p^{\prime}(.)}(\Omega)\right\} .
\end{aligned}
$$


By Lemma 2.4,the quadruple

$$
\left(\begin{array}{cc}
W_{0}^{m, p(.)}(\Omega) & W^{-m, p^{\prime}(.)}(\Omega) \\
H_{0}^{m, p(.)}(\Omega) & H^{-m, p^{\prime}(.)}(\Omega)
\end{array}\right)
$$

forms a complementary system.

We say that a sequence $\left\{u_{n}\right\} \subset W^{-m, p^{\prime}(\cdot)}(\Omega)$ converges to $u \in W^{-m, p^{\prime}(.)}(\Omega)$ in the modular sense, denote $u_{n} \rightarrow u(\bmod )$ in $W^{-m, p^{\prime}(.)}$, if $u_{n}$ and $u$ have representations

$$
u_{n}=\sum_{|\alpha| \leq m}(-1)^{|\alpha|} D^{\alpha} g_{\alpha}^{(n)}, u=\sum_{|\alpha| \leq m}(-1)^{|\alpha|} D^{\alpha} g_{\alpha},
$$

such that $g_{\alpha}^{(n)}, g_{\alpha} \in L^{p^{\prime}(.)}(\Omega)$ and $g_{\alpha}^{(n)} \rightarrow g_{\alpha}(\bmod )$ in $L^{p^{\prime}(.)}$ for all $|\alpha| \leq m$.

Let $A$ be a subset of a Generalized Sobolev Space $Y$. We denote by $\bar{A}^{\text {mod }}$ the sequentiel modular closure of $A$,i.e.

$$
\bar{A}^{\text {mod }}=\left\{u \in Y / \text { there exists }\left\{u_{n}\right\} \subset A \text { such that } u_{n} \rightarrow u(\bmod ) \text { in } Y\right\} .
$$

\subsection{Some classes of mappings of monotone type. Let}

$$
\left(\begin{array}{cc}
Y & Z \\
Y_{0} & Z_{0}
\end{array}\right)=\left(\begin{array}{cc}
W_{0}^{m, p(.)}(\Omega) & W^{-m, p^{\prime}(.)}(\Omega) \\
H_{0}^{m, p(.)}(\Omega) & H^{-m, p^{\prime}(.)}(\Omega)
\end{array}\right)
$$

be a complementary system formed of Generalized Sobolev Spaces in which $\Omega \subset \mathbb{R}^{N}$ is open, bounded and satisfies the segment property and $p \in \mathcal{P}^{\log }(\Omega)$ satisfy $1<p^{\prime-} \leq p^{\prime+} \leq \infty$. We consider mappings $F: D_{F} \rightarrow Z$ which satisfy the following conditions:

(i): $Y_{0} \subset D_{F} \subset Y$,

(ii): $F$ is finitely continuous, i.e., the restriction of the mapping $F$ to any finite dimensional supspace $X \subset Y_{0}$ is continuous from the topology of $X$ to the weak topology of $Z$.

We shall next define some classes of mappings of monotone type:

(i): $F$ is bounded, denote $F \in(B D)$, if the set $F(A) \subset Z$ is bounded when $A \subset D_{F}$ is bounded.

(ii): $F$ is strongly quasibounded, denote $F \in(Q B)$, if the conditions

$\left\{u_{n}\right\} \subset D_{F}$ bounded and $\left\langle F\left(u_{n}\right), u_{n}-\bar{u}\right\rangle$ is bounded from above for some $\bar{u} \in Y_{0}$ imply that $\left\{F\left(u_{n}\right)\right\}$ is bounded in $Z$.

(iii): $F$ is continuous, denote $F \in(C O N T)$, if the conditions $\left\{u_{n}\right\} \subset D_{F}$, $u \in D_{F}$ and $\left\|u_{n}-u\right\|_{Y} \rightarrow 0$ imply that $\left\|F\left(u_{n}\right)-F(u)\right\|_{Z} \rightarrow 0$.

(iv): $F$ is of the class $\left(S_{+}\right)$, denote $F \in\left(S_{+}\right)$,if the conditions $\left\{u_{n}\right\} \subset D_{F}, u_{n} \rightarrow u \in Y$ in $Y$ and $\limsup _{n \rightarrow \infty}\left\langle F\left(u_{n}\right), u_{n}-u\right\rangle \leq 0$ imply that $u \in D_{F}$, and $\left\|u_{n}-u\right\|_{Y} \rightarrow 0$.

(v): $F$ is demicontinuous if the conditions $\left\{u_{n}\right\} \subset D_{F}, u \in D_{F}$ and $u_{n} \rightarrow u$ imply that $F\left(u_{n}\right) \rightarrow F(u)$.

(vi): $F$ is pseudomonotone, $F \in(P M)$, if the conditions

$\left\{u_{n}\right\} \subset D_{F}, u_{n} \rightarrow u$ in $Y, F\left(u_{n}\right) \rightarrow \chi$ in $Z$ and limsup $_{n \rightarrow \infty}\left\langle F\left(u_{n}\right), u_{n}\right\rangle \leq\langle\chi, u\rangle$

imply that $u \in D_{F}, \chi=F(u)$ and $\left\langle F\left(u_{n}\right), u_{n}\right\rangle \rightarrow\langle F(u), u\rangle$. 
(vi): $F$ is of the class (MOD), denote $F \in(M O D)$, if the conditions

$\left\{u_{n}\right\} \subset D_{F}, u_{n} \rightarrow u$ in $Y, F\left(u_{n}\right) \rightarrow \chi$ in $Z$ and

$\limsup _{n \rightarrow \infty}\left\langle F\left(u_{n}\right), u_{n}\right\rangle \leq\langle\chi, u\rangle$

imply that $u \in D_{F}, \chi=F(u)$ and there exists a subsequence $\left\{u_{n^{\prime}}\right\}$ such that $u_{n^{\prime}} \rightarrow u$ (mod) in $Y$ and $F\left(u_{n^{\prime}}\right) \rightarrow F(u)$ (mod) in $Z$.

\section{Degree theory in Generalized Sobolev spaces}

\subsection{An outline of Brouwers degree theory.}

Theorem 3.1. Let $X=\mathbb{R}^{n}=Y$ for a given positive integer $n$. For bounded open subsets $G$ of $X$, consider continuous mappings $f: \bar{G} \rightarrow Y$ and points $y_{0}$ in $Y$ such that $y_{0} \notin f(\partial G)$. Then to each such triple $\left(f, G, y_{0}\right)$, there corresponds an integer $d\left(f, G, y_{0}\right)$ having the following properties:

(a): Existence: if $d\left(f, G, y_{0}\right) \neq 0$, then $y_{0} \in f(G)=$,

(b): Additivity: if $f: \bar{G} \rightarrow Y$ is a continuous map with $G$ a bounded open set in $X$ and $G_{1}, G_{2}$ are a pair of disjoint open subsets of $G$ such that $y_{0} \notin f\left(\bar{G} \backslash\left(G_{1} \cup G_{2}\right)\right)$, then

$$
d\left(f, G, y_{0}\right)=d\left(f, G_{1}, y_{0}\right)+d\left(f, G_{2}, y_{0}\right),
$$

$(c)$ : Invariance under homotopy: Let $G$ be a bounded open set in $X$, and consider a continuous homotopy $\left\{f_{t}: 0 \leq t \leq 1\right\}$ of maps of $\bar{G}$ into $Y$. $\left\{y_{t}: 0 \leq t \leq 1\right\}$ be a continuous curve in $Y$ such that $y_{t} \notin f_{t}(\partial G)$ for any $t \in[0,1]$, then $d\left(f_{t}, G, y_{t}\right)$ is constant in $t$ on $[0,1]$,

$(d)$ : Normalization: If $f_{0}$ is the identity map of $X$ onto $Y$, then for every bounded open $G$ and $y_{0} \in f_{0}(G)$ then

$$
d\left(f_{0}, G, y_{0}\right)=1 \text {. }
$$

Theorem 3.2. The degree function $d(f, G, y 0)$ is uniquely determined by the four conditions of Theorem 3.1.

Remark 3.3. Theorem 3.1 is an appropriately formalized version of the properties of the classical Brouwer degree. Theorem 3.2 contains an observation made independently in 1972 and 1973 by Fuhrer [9] and Amann and Weiss [17], respectively.

\subsection{Construction of a degree function in Generalized Sobolev Spaces.}

Let

$$
\left(\begin{array}{cc}
Y & Z \\
Y_{0} & Z_{0}
\end{array}\right)=\left(\begin{array}{cc}
W_{0}^{m, p(.)}(\Omega) & W^{-m, p^{\prime}(.)}(\Omega) \\
H_{0}^{m, p(.)}(\Omega) & H^{-m, p^{\prime}(\cdot)}(\Omega)
\end{array}\right)
$$

be a complementary system formed of Generalized Sobolev Spaces in which

$\Omega \subset \mathbb{R}^{N}$ is open, bounded and satisfies the segment property and $p \in \mathcal{P}^{\log }(\Omega)$ satisfy $1<{p^{\prime}}^{-} \leq p^{\prime+} \leq \infty$. We define the class $\mathcal{F}$ of admissible mappings and the class $\mathcal{H}$ of admissible homotopies as follows:

$F: D_{F} \subset Y \rightarrow Z$ belongs to $\mathcal{F}$, if

(a): $F$ is a strongly quasibounded mapping of the class $(M O D)$.

$F: D_{F} \subset Y \rightarrow Z$ belongs to $\mathcal{F}^{a}$, if $F \in \mathcal{F}$ and the following conditions hold:

(b): if $\left\{u_{n}\right\} \subset D_{F}$ is bounded, $t_{n} \rightarrow 0^{+}$and $\left\langle t_{n} F\left(u_{n}\right), u_{n}-\bar{u}\right\rangle$ is bounded from above for some $\bar{u} \in Y$, then $\left\{t_{n} F\left(u_{n}\right)\right\} \subset Z$ is bounded, 
(c): if $\left\{u_{n}\right\} \subset D_{F}, u_{n} \rightarrow u \in Y$ for $\sigma\left(Y, Z_{0}\right), t_{n} \rightarrow 0^{+}, t_{n} F\left(u_{n}\right) \rightarrow \chi \in Z$ for $\sigma\left(Z, Y_{0}\right)$ and $\limsup \left\langle t_{n} F\left(u_{n}\right), u_{n}\right\rangle \leq\langle\chi, u\rangle$, then $\left\langle t_{n} F\left(u_{n}\right), u_{n}\right\rangle \rightarrow\langle\chi, u\rangle$,

(d): if $\left\{u_{n}\right\} \subset D_{F}, u_{n} \rightarrow u$ (mod) in $Y, t_{n} \rightarrow 0^{+}$, $t_{n} F\left(u_{n}\right) \rightarrow \chi \in Z\left(\sigma\left(Z, Y_{0}\right)\right)$ in $Z$ and $\limsup \left\langle t_{n} F\left(u_{n}\right), u_{n}\right\rangle \leq\langle\chi, u\rangle$, then $t_{n} F\left(u_{n}\right) \rightarrow 0$ (mod) in $Z$.

The homotopy $H: D_{H} \rightarrow Z$ belongs to $\mathcal{H}$, if $H$ is a strongly quasibounded homotopy of the class $(M O D)$.

Lemma 3.4. If $F, G \in \mathcal{F}^{a}$, then $H(t, u)=t F(u)+(1-t) G(u)$ belongs to $\mathcal{H}$ with

$$
D_{H_{t}}=\left\{\begin{array}{c}
D_{F} \cap D_{G}, \text { if } 0<t<1 \\
D_{G}, \text { if } t=0 \\
D_{F}, \text { if } t=1 .
\end{array}\right.
$$

Proof. Step 1

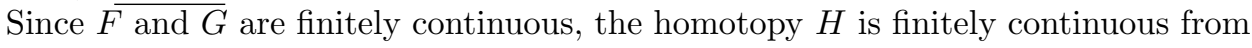
the norm topology of $[0,1] \times Y$ to the weak topology of $Z$.

\section{Step 2}

We shall prove that $H$ is strongly quasibounded.

Assume $\left\{t_{n}\right\} \subset[0,1],\left\{u_{n}\right\} \subset D_{H_{t_{n}}}$ and $\left\langle H\left(t_{n}, u_{n}\right), u_{n}-\bar{u}\right\rangle$ is bounded from above for some $\bar{u} \in Y_{0}$. It follows that $\left\langle t_{n^{\prime}} F\left(u_{n^{\prime}}\right), u_{n^{\prime}}-\bar{u}\right\rangle$ or $\left\langle\left(1-t_{n^{\prime}}\right) G\left(u_{n^{\prime}}\right), u_{n^{\prime}}-\bar{u}\right\rangle$ is bounded from above for a subsequence.

We may also suppose that $\left\langle t_{n^{\prime}} F\left(u_{n^{\prime}}\right), u_{n^{\prime}}-\bar{u}\right\rangle$ is bounded from above. By condition (b) and the fact that $F$ is strongly quasibounded, the sequence $\left\{t_{n^{\prime}} F\left(u_{n^{\prime}}\right)\right\}$ is bounded in $Z$. Consequently, $\left\langle t_{n^{\prime}} F\left(u_{n^{\prime}}\right), u_{n^{\prime}}-\bar{u}\right\rangle$ is bounded implying that $\langle(1-$ $\left.\left.t_{n^{\prime}}\right) G\left(u_{n^{\prime}}\right), u_{n^{\prime}}-\bar{u}\right\rangle$ is bounded from above. Therefore $\left\{\left(1-t_{n^{\prime}}\right) G\left(u_{n^{\prime}}\right)\right\}$ is also bounded in $Z$ and hence $\left\{H\left(t_{n^{\prime}}, u_{n^{\prime}}\right)\right\}$ is bounded in $Z$.

By contradiction argument, $\left\{H\left(t_{n}, u_{n}\right)\right\}$ is bounded in $Z$.

Step 3

We shall next prove that $H$ is a homotopy of the class $(M O D)$.

Asuume that $t_{n} \subset[0,1], t_{n} \rightarrow t \in[0,1],\left\{u_{n}\right\} \subset D_{H_{t_{n}}}, u_{n} \rightarrow u$ in $Y, H\left(t_{n}, u_{n}\right) \rightarrow \chi$ in $Z$ and limsup $_{n \rightarrow \infty}\left\langle H\left(t_{n}, u_{n}\right), u_{n}\right\rangle \leq\langle\chi, u\rangle$.

Deducing as above, $\left\{t_{n^{\prime}} F\left(u_{n^{\prime}}\right)\right.$ and $\left\{\left(1-t_{n^{\prime}}\right) G\left(u_{n^{\prime}}\right)\right\}$ are bounded in $Z$ for some subsequence. We may assume that $t_{n^{\prime}} F\left(u_{n^{\prime}}\right) \rightarrow \chi_{1}$ and $\left(1-t_{n^{\prime}}\right) G\left(u_{n^{\prime}}\right) \rightarrow \chi_{2}$ in $Z$. It is clear that $\chi=\chi_{1}+\chi_{2}$. We may assume that limsup $_{n \rightarrow \infty}\left\langle t_{n^{\prime}} F\left(u_{n^{\prime}}\right), u_{n^{\prime}}\right\rangle \leq$ $\left\langle\chi_{1}, u\right\rangle$ or limsup $_{n \rightarrow \infty}\left\langle\left(1-t_{n^{\prime}}\right) G\left(u_{n^{\prime}}\right), u_{n^{\prime}}\right\rangle \leq\left\langle\chi_{2}, u\right\rangle$. Suppose, for example, that

$$
\text { limsup }_{n \rightarrow \infty}\left\langle t_{n^{\prime}} F\left(u_{n^{\prime}}\right), u_{n^{\prime}}\right\rangle \leq\left\langle\chi_{1}, u\right\rangle .
$$

By condition (c) and the fact that $F$ belongs to the class $(M O D)$, we have $\left\langle t_{n^{\prime}} F\left(u_{n^{\prime}}\right), u_{n^{\prime}}\right\rangle \rightarrow$ $\left\langle\chi_{1}, u\right\rangle$. Hence

$$
\limsup _{n \rightarrow \infty}\left\langle\left(1-t_{n^{\prime}}\right) G\left(u_{n^{\prime}}\right), u_{n^{\prime}}\right\rangle \leq\left\langle\chi_{2}, u\right\rangle .
$$

If $0<t<1$, then $F\left(u_{n^{\prime}}\right) \rightarrow \frac{\chi_{1}}{t}$ in $Z, G\left(u_{n^{\prime}}\right) \rightarrow \frac{\chi_{2}}{1-t}$ in $Z,\left\langle F\left(u_{n^{\prime}}\right), u_{n^{\prime}}\right\rangle \rightarrow\left\langle\frac{1}{t} \chi_{1}, u\right\rangle$ and $\limsup _{n \rightarrow \infty}\left\langle G\left(u_{n^{\prime}}\right), u_{n^{\prime}}\right\rangle \leq\left\langle\frac{1}{1-t} \chi_{2}, u\right\rangle$.

Therefore $u \in D_{F} \cap D_{G}, \frac{\chi_{1}}{t}=F(u), \frac{\chi_{2}}{1-t}=G(u), u_{n^{\prime}} \rightarrow u(\bmod )$ in $Y$ and $F\left(u_{n^{\prime}}\right) \rightarrow$ $F(u), G\left(u_{n^{\prime}}\right) \rightarrow G(u)(\mathrm{mod})$ in $Z$. Hence $u \in D_{H_{t}}$ and $H\left(t_{n^{\prime}}, u_{n^{\prime}}\right) \rightarrow H(t, u)(\mathrm{mod})$ in $Z$. If $t=0$, then $G\left(u_{n^{\prime}}\right) \rightarrow \frac{\chi_{2}}{1-t}$ in $Z$ and $\limsup _{n \rightarrow \infty}\left\langle G\left(u_{n^{\prime}}\right), u_{n^{\prime}}\right\rangle \leq\left\langle\frac{1}{1-t} \chi_{2}, u\right\rangle$. Therefore $u \in D_{G}, u_{n^{\prime}} \rightarrow u(\mathrm{mod})$ in $Y$ and $G\left(u_{n^{\prime}} \rightarrow G(u)(\mathrm{mod})\right.$ in $Z$. Moreover, by condition (d), we have $t_{n^{\prime}} F\left(u_{n^{\prime}}\right) \rightarrow 0$ in the modular sense. Hence $u \in D_{H_{0}}$ and $H\left(t_{n^{\prime}}, u_{n^{\prime}}\right) \rightarrow H(t, u)(\mathrm{mod})$ in $Z$. If $t=1$, we make an analogue deduction to 
obtain $u \in D_{F}=D_{H_{1}}$ and $u_{n^{\prime}} \rightarrow u(\bmod )$ in $Y$ and $F\left(u_{n^{\prime}}\right) \rightarrow F(u)(\bmod )$ in $Z$. Consequently, $H$ is a homotopy of the class $(M O D)$.

Our aim in this subsection is to construct an integer-valued degree function $d(F, G, f)$ for $F \in \mathcal{F}, G \subset Y_{0}$ open and bounded in $Y_{0}, f \in Z_{0}$ and $f \notin F\left({\overline{\partial_{Y_{0}}}}^{\text {mod }}\right) \cap$ ${\overline{F\left(\partial_{Y_{0}} G\right.}}^{\text {mod }}$ satisfying the following conditions:

$\left(C_{1}\right)$ : Existence: if $d(F, G, f) \neq 0$, then $f \in F\left(\bar{G}^{\text {mod }}\right) \cap \overline{F(G)}^{\text {mod }}$,

$\left(C_{2}\right)$ : Additivity: if $G_{1}, G_{2} \subset G$ are open and bounded, $G_{1} \cap G_{2}=\emptyset$ and $f \notin F\left({\overline{\bar{G} \backslash\left(G_{1} \cup G_{2}\right)}}^{\text {mod }}\right) \cap{\overline{F\left(\bar{G} \backslash\left(G_{1} \cup G_{2}\right)\right)}}^{\text {mod }}$, then

$$
d(F, G, f)=d\left(F, G_{1}, f\right)+d\left(F, G_{2}, f\right),
$$

$\left(C_{3}\right)$ : Homotopy invariance: if $H \in \mathcal{H}, f \in Z_{0}$ and $f \notin H\left([0,1] \times{\overline{\partial_{Y_{0}}}}^{\text {mod }}\right) \cap$ $\overline{H\left([0,1] \times \partial_{Y_{0}} G\right)}{ }^{\text {mod }}$, then

$$
d(H(t, \cdot), G, f)=\text { constant for all } t \in[0,1],
$$

$\left(C_{4}\right)$ : Normalization: There exists a normalising map $K \in \mathcal{F}^{a}$ such that if $f \in Z_{0}, f \notin K\left({\overline{\partial_{Y_{0}} G}}^{\text {mod }}\right) \cap{\overline{K\left(\partial_{Y_{0}} G\right)}}^{\text {mod }}$ and $f \in K(G)$, then

$$
d(K, G, f)=1 .
$$

Remark 3.5. We shall always assume in the applications that $1<p^{-} \leq p(\cdot) \leq p^{+}<\infty$. This restriction means that instad of modular closure we have norm closures. In these cases the corresponding degree theories can be formulated as fellows:

$F \in \mathcal{F}, G \subset Y$ open and bounded in $Y, f \in Z_{0}$ and $f \notin F\left(\partial_{Y} G\right)$

$\left(c_{1}\right)$ : Existence: if $d(F, G, f) \neq 0$, then $f \in F(G)$,

$\left(c_{2}\right)$ : Additivity: if $G_{1}, G_{2} \subset G$ are open and bounded, $G_{1} \cap G_{2}=\emptyset$ and $f \notin F\left(\bar{G} \backslash\left(G_{1} \cup G_{2}\right)\right)$, then

$$
d(F, G, f)=d\left(F, G_{1}, f\right)+d\left(F, G_{2}, f\right),
$$

$\left(c_{3}\right)$ : Homotopy invariance: if $H \in \mathcal{H}, f \in Z_{0}$ and $f \notin H\left([0,1] \times \partial_{Y} G\right)$, then

$$
d(H(t, \cdot), G, f)=\text { constant for all } t \in[0,1],
$$

$\left(c_{4}\right)$ : Normalization: There exists a normalising map $K \in \mathcal{F}^{a}$ such that if $f \in Z_{0}, f \notin K\left(\partial_{Y} G\right)$ and $f \in K(G)$, then

$$
d(K, G, f)=1 .
$$

For the construction of such degree, we need the following:

Definition 3.6. Let $\Lambda$ be the set of all finite dimensional subspaces of $Y_{0}$.

Denote

$Y_{0}=X_{\lambda} \oplus Y_{\lambda}$ for all $X_{\lambda} \in \Lambda$, where $Y_{\lambda}$ is the closed complement of $X_{\lambda}([14, \mathrm{p} .157])$,

$A_{\lambda}=A \cap X_{\lambda}$, when $A \subset Y$,

$P_{\lambda}: Y_{0} \rightarrow X_{\lambda}$ the projection map,

$P_{\lambda}^{*}: X_{\lambda} \rightarrow Z,\left\langle P_{\lambda}^{*}(u), v\right\rangle=\left\langle u, P_{\lambda}(v)\right\rangle$ for all $u \in X_{\lambda}$ and $v \in Y_{0}$.

For the natural injection $\phi_{\lambda}: X_{\lambda} \rightarrow Y_{0}$, we define

$\phi_{\lambda}^{*}: Z \rightarrow X_{\lambda},\left\langle\phi_{\lambda}^{*}(u), v\right\rangle=\left\langle u, \phi_{\lambda}(v)\right\rangle$ for all $u \in Z$ and $v \in X_{\lambda}$.

If $F: Y_{0} \rightarrow Z$, then $F_{\lambda}: X_{\lambda} \rightarrow X_{\lambda}, F_{\lambda}(x)=\phi_{\lambda}^{*}\left(F\left(\phi_{\lambda}(x)\right)\right)$.

Let $d_{n}$ be the Brouwer degree for continuous maps from $\mathbb{R}^{n}$ to $\mathbb{R}^{n}$. 
Lemma 3.7. [5] Let $G \subset \mathbb{R}^{n}$ be open and bounded and $F: \bar{G} \subset \mathbb{R}^{n} \rightarrow \mathbb{R}^{n}$ continuous such that $0 \notin F(\partial G)$. Define a mapping

$$
\begin{gathered}
F^{\prime}: \bar{G} \times[-1,1]^{m} \subset \mathbb{R}^{n+m} \rightarrow \mathbb{R}^{n+m} \\
F^{\prime}(x, y)=(F(x), y) \text { for all } x \in \bar{G}, y \in[-1,1]^{m} .
\end{gathered}
$$

Then

$$
d_{n}(F, G, 0)=d_{n+m}\left(F^{\prime}, G \times(-1,1)^{m}, 0\right) .
$$

Let $d_{\lambda}$ be the Brouwer degree in the space $X_{\lambda}$.

Lemma 3.8. Let $G \subset Y_{0}$ be open and bounded in $Y_{0}, X_{\lambda} \subset X_{\mu} \in \Lambda$, such that $G \cap X_{\lambda} \neq \emptyset$ and $F \in(M O D)$. If $d_{\lambda}\left(F_{\lambda}, G_{\lambda}, 0\right) \neq d_{\mu}\left(F_{\mu}, G_{\mu}, 0\right)$ or one of the degrees is not defined, then there exists $u \in \partial_{Y_{0}} G$ such that

$$
\langle F(u), u\rangle \leq 0 \text { and }\langle F(u), v\rangle=0 \text { for all } v \in X_{\lambda} .
$$

Proof. If one of the degrees $d_{\lambda}\left(F_{\lambda}, G_{\lambda}, 0\right)$ and $d_{\mu}\left(F_{\mu}, G_{\mu}, 0\right)$ is not defined, there exists $u \in \partial_{X_{\lambda}} G_{\lambda} \subset \partial_{Y_{0}} G$ such that $F_{\lambda}(u)=0$ or $u \in \partial_{X_{\mu}} G_{\mu} \subset \partial_{Y_{0}} G$ such that $F_{\mu}(u)=0$. In both cases the proof is complete.

Otherwise we define a continuous mapping $S: X \mu \rightarrow X_{\mu}$,

$$
S(x)=\phi_{\lambda}^{*}\left(F\left(P_{\lambda}(x)\right)\right)+x-P_{\lambda}(x .)
$$

By Lemma 3.7 we have

$$
d_{\lambda}\left(F_{\lambda}, G_{\lambda}, 0\right)=d_{\mu}\left(S, G_{\lambda} \times(-1,1)^{m}, 0\right),
$$

where $m=\operatorname{dim} X_{\mu}-\operatorname{dim} X_{\lambda}$. If $S(x)=0$ for some $x \in G_{\mu}$, then $x-P_{\lambda}(x)=0$, implying $x \in G_{\lambda} \times(-1,1)^{m}$. If $S(x)=0$ for some $x \in G_{\lambda} \times(-1,1)^{m}$ then $x-P_{\lambda}(x)=0$, which means that $x \in G_{\lambda} \subset G_{\mu}$. By the excision property of the Brouwer degree, we have

$$
d_{\mu}\left(S, G_{\mu}, 0\right)=d_{\mu}\left(S, G_{\lambda} \times(-1,1)^{m}, 0\right) .
$$

Hence

$$
d_{\mu}\left(F_{\mu}, G_{\mu}, 0\right) \neq d_{\mu}\left(S, G_{\mu}, 0\right) .
$$

Define another mapping $S^{\prime}: X \mu \rightarrow X_{\mu}$,

$$
\left.S^{\prime}(x)=\phi_{\lambda}^{*}(F x)\right)+x-P_{\lambda}(x) .
$$

We shall prove that $d_{\mu}\left(S, G_{\mu}, 0\right)=d_{\mu}\left(S^{\prime}, G_{\mu}, 0\right)$. Consider the homotopy

$$
H(t, u)=t S(u)+(1-t) S^{\prime}(u)=u-P_{\lambda}(u)+\phi_{\lambda}^{*}\left[t F\left(P_{\lambda}(u)\right)+(1-t) F(u)\right] .
$$

If $H(t, u)=0$ for some $0 \leq t \leq 1$ and $u \in \bar{G}_{\mu}^{X_{\mu}}$, then $u=P_{\lambda}(u) \in X_{\lambda}$ implying $\phi_{\lambda}^{*}(F(u))=F_{\lambda}(u)=0$ and therefore $u \in G_{\lambda} \subset G_{\mu}$. By homoyopy invariance, we have

$$
d_{\mu}\left(S, G_{\mu}, 0\right)=d_{\mu}\left(S^{\prime}, G_{\mu}, 0\right)
$$

We can thus deduce that

$$
d_{\mu}\left(F_{\mu}, G_{\mu}, 0\right) \neq d_{\mu}\left(S^{\prime}, G_{\mu}, 0\right) .
$$

Consider the homotopy $H:[0,1] \times X_{\mu} \rightarrow X_{\mu}$,

$$
H(t, u)=t S^{\prime}(u)+(1-t) F_{\mu}(u) .
$$


By the homotopy invariance of the Brouwer degree, $H(t, u)=0$ for some $u \in \partial_{X_{\mu}} G_{\mu} \subset \partial_{Y} G$ and $t \in(0,1)$. Let $v \in X_{\lambda}$ be arbitrary, then

$$
\begin{aligned}
\langle H(t, u), v\rangle & =t\left\langle S^{\prime}(u), v\right\rangle+(1-t)\left\langle F_{\lambda}(u), v\right\rangle \\
& =t\langle F(u), v\rangle+t\left\langle u-P_{\lambda}(u), v\right\rangle+(1-t)\langle F(u), v\rangle \\
& =t\langle F(u), v\rangle+(1-t)\langle F(u), v\rangle=0,
\end{aligned}
$$

implying $\langle F(u), v\rangle=0$. Moreover,

$$
\begin{aligned}
\left\langle H(t, u), u-P_{\lambda}(u)\right\rangle & \left.=t\left\langle\phi_{\lambda}^{*}(F(u)), u-P_{\lambda}(u)\right\rangle+t\left\langle u-P_{\lambda}(u)\right), u-P_{\lambda}(u)\right\rangle \\
& +(1-t)\left\langle F_{\lambda}(u), u-P_{\lambda}(u)\right\rangle \\
& =t\left\|u-P_{\lambda}(u)\right\|^{2}+(1-t)\langle F(u), u\rangle=0 .
\end{aligned}
$$

Hence $\langle F(u), u\rangle \leq 0$.

Lemma 3.9. Let $H: D_{H} \rightarrow Z$ be a strongly quasibounded (MOD) homotopy and $A \subset Y_{0}$ closed and bounded. If

$$
0 \notin H\left([0,1] \times \bar{A}^{\text {mod }}\right) \cap \overline{H([0,1] \times A)}^{\text {mod }},
$$

then there exists $X_{\lambda} \in \Lambda$ such that

$$
0 \notin H_{\mu}\left([0,1] \times A_{\mu}\right) \text { for all } X \mu \supset X_{\lambda} .
$$

Proof. By contradiction, suppose that

$$
\forall X_{\lambda} \in \Lambda, \exists X \mu \supset X_{\lambda}, \exists\left(t_{\mu}, a_{\mu}\right) \in[0,1] \times A_{\mu} ; H_{\mu}\left(t_{\mu}, a_{\mu}\right)=0 .
$$

Define a set

$$
V_{\lambda}=\left\{(t, a) \in[0,1] \times A \mid\langle H(t, a), a\rangle \leq 0 \text { and }\langle H(t, a), v\rangle=0 \text { for all } v \in X_{\lambda}\right\},
$$

which is non-empty for all $\lambda$. If $X_{\lambda_{1}}, X_{\lambda_{2}}, \ldots, X_{\lambda_{n}} \in \Lambda$ and if $\cup_{i=1}^{n} X_{\lambda_{i}} \subset X_{\lambda}$, then

$$
V_{\lambda} \subset \cap_{i=1}^{n} V_{\lambda_{i}} \text {. }
$$

Therefore the family $\left\{V_{\lambda}\right\}$ has the finite intersection property. Denote $\tau$ the topology $\|.\|_{\mathbb{R}} \times \sigma\left(Y, Z_{0}\right)$. The set ${\overline{V_{\lambda}}}^{\tau}$ is bounded and $\tau$-closed, which implies by Alaoglu's Theorem that it is $\tau$-compact. Hence

$$
\cap{\overline{V_{\lambda}}}^{\tau} \neq \emptyset \text {. }
$$

Choose $\left(t_{0}, u_{0}\right) \in \cap{\overline{V_{\lambda}}}^{\tau}$. The space $Y_{0}$ is separable, we may denote $Y_{0}=\overline{\left\{y_{1}, y_{2}, \ldots\right\}}$. Define $X_{\lambda_{i}}=\operatorname{sp}\left\{y_{1}, y_{2}, \ldots, y_{i}\right\}$, then we have $X_{\lambda_{1}} \subset X_{\lambda_{2}} \subset \ldots \subset X_{\lambda_{i}} \subset \ldots$ The space $Z_{0}$ is separable, then the $\tau$-topology in the set $\overline{[0,1]} \tau=A$ is metrizable ([18, p.782]. Denote $d_{\tau}$ this metric. For every $i=1,2, \ldots$, we have $\left(t_{0}, u_{0}\right) \in{\overline{V_{\lambda_{i}}}}^{\tau}$, then we can choose a sequence $\left\{\left(t_{n}^{(i)}, u_{n}^{(i)}\right)\right\}_{n} \subset V_{\lambda_{i}}$ such that

$$
(\forall i \in \mathbb{N}), d_{\tau}\left\{\left(t_{n}^{(i)}, u_{n}^{(i)}\right),\left(t_{0}, u_{0}\right)\right\}<\frac{1}{n} .
$$

Therefore $\left(t_{n}^{(n)}, u_{n}^{(n)}\right) \rightarrow\left(t_{0}, u_{0}\right)$ with respect to $\tau$ and

$$
\left\langle H\left(t_{n}^{(n)}, u_{n}^{(n)}\right), u_{n}^{(n)}\right\rangle \leq 0,
$$

$$
\left\langle H\left(t_{n}^{(n)}, u_{n}^{(n)}\right), v\right\rangle=0 \text { for all } v \in X_{\lambda_{n}} .
$$


Since $H$ is strongly quasibounded, the sequence $\left\{H\left(t_{n}^{(n)}, u_{n}^{(n)}\right)\right\}$ is bounded in $Z$ by (3.1). Therefore we can choose a subsequence $\left\{H\left(t_{n^{\prime}}^{\left(n^{\prime}\right)}, u_{n^{\prime}}^{\left(n^{\prime}\right)}\right)\right\}$ such that $H\left(t_{n^{\prime}}^{\left(n^{\prime}\right)}, u_{n^{\prime}}^{\left(n^{\prime}\right)}\right) \rightarrow z \in Z$. Moreover, by (3.2), we have

$$
\langle\chi, v\rangle=0 \text { for all } v \in \cup_{n=1}^{\infty} X_{\lambda_{n}} .
$$

Because $\cup_{n=1}^{\infty} X_{\lambda_{n}}$ is norm-dense in $Y_{0}$, we have

$$
\langle\chi, v\rangle=0 \text { for all } v \in Y_{0} \text {. }
$$

Therefore $\chi=0$ as an element of the dual space $Z$. Moreover, $Y_{0}$ is $\sigma(Y, Z)$-dense in $Y$, which implies that

$$
\langle\chi, v\rangle=0 \text { for all } v \in Y \text {. }
$$

Consequently, we have

$$
\limsup \left\langle H\left(t_{n^{\prime}}^{\left(n^{\prime}\right)}, u_{n^{\prime}}^{\left(n^{\prime}\right)}\right), u_{n^{\prime}}^{\left(n^{\prime}\right)}\right\rangle \leq 0=\left\langle\chi, u_{0}\right\rangle .
$$

Since $H$ is a $(M O D)$ homotopy, we have $u_{0} \in D_{H_{t}}, u_{n^{\prime}}^{\left(n^{\prime}\right)} \rightarrow u_{0}(\bmod )$ in $Y$ and $H\left(t_{n^{\prime}}^{\left(n^{\prime}\right)}, u_{n^{\prime}}^{\left(n^{\prime}\right)}\right) \rightarrow H\left(t_{0}, u_{0}\right)=0(\bmod )$ in $Z$ for a subsequence. Therefor

$$
0 \in H\left([0,1] \times \bar{A}^{\text {mod }}\right) \cap \overline{H([0,1] \times A)}^{\text {mod }},
$$

which is a contradiction.

The next lemma proves that the degree $d_{\mu}$ will stabilize when we go to the limit.

Lemma 3.10. Let $F \in(M O D)$ be a strongly quasibounded and $G \subset Y_{0}$ open and bounded in $Y_{0}$. If

$$
0 \notin F\left({\overline{\partial_{Y_{0}} G}}^{\text {mod }}\right) \cap{\overline{F\left(\partial_{Y_{0}} G\right)}}^{\text {mod }},
$$

then there exists $X_{\lambda} \in \Lambda$ such that

$$
0 \notin F_{\mu}\left(\partial_{X_{\mu}} G_{\mu}\right) \text { and } d_{\mu}\left(F_{\mu}, G_{\mu}, 0\right)=\text { constant for every } X_{\mu} \supset X_{\lambda} .
$$

Proof. The first part follows immediately from Lemma 3.9.

For the second part, suppose, by contradiction, that

$$
\forall X_{\lambda} \in \Lambda, \exists X_{\mu} \supset X_{\lambda} ; d_{\lambda}\left(F_{\lambda}, G_{\lambda}, 0\right) \neq d_{\mu}\left(F_{\mu}, G_{\mu}, 0\right) .
$$

By Lemma 3.8, there exists $u_{\lambda} \in \partial_{Y_{0}} G$ such that

$$
\left\langle F\left(u_{\lambda}\right), u_{\lambda}\right\rangle \leq 0 \text { and }\left\langle F\left(u_{\lambda}\right), v\right\rangle=0 \text { for every } v \in X_{\lambda} .
$$

Define a set

$$
\left.V_{\lambda}=u \in \partial_{Y_{0}} G \mid\langle F(u), u\rangle \leq 0 \text { and }\langle F(u), v\rangle=0 \text { for all } v \in X_{\lambda}\right\},
$$

which is non-empty. As in the proof of Lemma 3.9, we can deduce the existence of $u_{0} \in \cap{\overline{V_{\lambda}}}^{\sigma\left(Y, Z_{0}\right)}$ and $\left\{u_{n}\right\} \subset \partial_{Y_{0}} G$ such that $u_{n} \rightarrow u_{0} \in D_{F}(\bmod )$ in $Y, F\left(u_{n}\right) \rightarrow$ $F\left(u_{0}\right)(\bmod )$ in $Z$ for a subsequence and $F\left(u_{0}\right)=0$.

Consequently, $0 \in F\left({\overline{\partial_{Y_{0}} G}}^{\text {mod }}\right) \cap{\overline{F\left(\partial_{Y_{0}} G\right)}}^{\text {mod }}$, which is a contradiction.

We can now define a degree function in the complementary system

$$
\left(\begin{array}{cc}
Y & Z \\
Y_{0} & Z_{0}
\end{array}\right)=\left(\begin{array}{cc}
W_{0}^{m, p(.)}(\Omega) & W^{-m, p^{\prime}(\cdot)}(\Omega) \\
H_{0}^{m, p(.)}(\Omega) & H^{-m, p^{\prime}(.)}(\Omega)
\end{array}\right)
$$


Definition 3.11. Let $F \in \mathcal{F}, G \subset Y_{0}$ open and bounded in $Y_{0}, f \in Z_{0}$ and $\left.f \notin F\left({\overline{\partial_{Y_{0}} G}}^{\text {mod }}\right) \cap \overline{F\left(\partial_{Y_{0}} G\right.}\right)^{\text {mod }}$. We then define

$$
d(F, G, f)=\lim _{\lambda} d_{\lambda}\left(F_{\lambda}-\phi_{\lambda}^{*}(f), G_{\lambda}, 0\right) .
$$

Theorem 3.12. The mapping $d$ in Definition 3.11 satisfies the conditions $\left(C_{1}\right)-$ $\left(C_{3}\right)$. Any mapping $K \in \mathcal{F}^{a}$ satisfying

$$
\langle K(u), u\rangle>0 \text {, when } u \neq 0 \text {, and } K(0)=0
$$

can be chosen as a normalising map.

Proof. It is enough to prove the conditions $\left(C_{1}\right)-\left(C_{3}\right)$ for $f=0$, because $F-f \in \mathcal{F}$, $H-f \in \mathcal{H}$ and

$$
d(F, G, f)=\lim _{\lambda} d_{\lambda}\left(F_{\lambda}-\phi_{\lambda}^{*}(f), G_{\lambda}, 0\right)=\lim _{\lambda} d_{\lambda}\left((F-f)_{\lambda}, G_{\lambda}, 0=d(F-f), G, 0\right) .
$$

$\left(C_{1}\right)$ If $d(F, G, 0) \neq 0$, then there exists $X_{\lambda} \in \Lambda$ such that $d_{\mu}\left(F_{\mu}, G_{\mu}, 0\right) \neq 0$ for all $X_{\mu} \supset X_{\lambda}$. Choose a sequence $\left\{v_{\mu_{n}}\right\}$ such that $v_{\mu_{n}} \in G_{\mu_{n}}, F_{\mu_{n}}\left(v_{\mu_{n}}\right)=0$, $\operatorname{dim} X_{\mu_{n}} \rightarrow \infty$ and $\cup X_{\mu_{n}}$ is dense in $Y_{0}$. Chose a subsequence $\left\{v_{\mu_{n^{\prime}}}\right\}$ such that $v_{\mu_{n^{\prime}}} \rightarrow v \in Y$. Since $\left\langle F\left(v_{\mu_{n^{\prime}}}\right), v_{\mu_{n^{\prime}}}\right\rangle=0$ and $F$ is strongly quasibounded, we have $F\left(v_{\mu_{n^{\prime}}}\right) \rightarrow \chi \in Z$ for a subsequence. We immediately see that $\langle\chi, w\rangle=0$ for all $w \in X_{\mu_{n}}$ for every $n$. Therefore, by density, $\langle\chi, w\rangle=0$ for all $w \in Y_{0}$, and by the $\sigma(Y, Z)$-density of $Y_{0}$ in the space $Y,\langle\chi, v\rangle=0$. Hence $\chi=0$ and

$$
\limsup \left\langle F\left(v_{\mu_{n^{\prime}}}\right), v_{\mu_{n^{\prime}}}\right\rangle=0=\langle\chi, v\rangle,
$$

implying $v_{\mu_{n^{\prime}}} \rightarrow v \in D_{F}(\mathrm{mod})$ in $Y$ and $F\left(v_{\mu_{n^{\prime}}}\right) \rightarrow F(v)(\mathrm{mod})$ in $Z$ for a further subsequence. Therefore $0 \in F\left(\bar{G}^{\text {mod }}\right) \cap \overline{F(G)}^{\text {mod }}$.

$\left(C_{2}\right)$ If $G_{1}, G_{2} \subset G$ are open and bounded in $Y_{0}, G_{1} \cap G_{2}=\emptyset$ and

$$
0 \notin F\left({\overline{\bar{G} \backslash\left(G_{1} \cup G_{2}\right)}}^{\text {mod }}\right) \cap{\overline{F\left(\overline{G \backslash\left(G_{1} \cup G_{2}\right)}\right.}}^{\text {mod }},
$$

then, by Lemma 3.10, there exists $X_{\lambda} \subset \Lambda$ such that

$$
0 \notin F_{\mu}\left(\bar{G}_{\mu} \backslash\left(G_{1, \mu} \cup G_{2, \mu}\right)\right) \text { for all } X_{\mu} \supset X_{\lambda} \text {. }
$$

Hence

$$
\begin{aligned}
d(F, G, 0) & =\lim _{\lambda} d_{\lambda}\left(F_{\lambda}, G_{\lambda}, 0\right) \\
& =\lim _{\lambda}\left[d_{\lambda}\left(F_{\lambda}, G_{1, \lambda}, 0\right)+d_{\lambda}\left(F_{\lambda}, G_{2, \lambda}, 0\right)\right] \\
& =d\left(F, G_{1}, 0\right)+d\left(F, G_{2}, 0\right) .
\end{aligned}
$$

$\left(C_{3}\right)$ Let $H \in \mathcal{H}$ and $G \subset Y_{0}$ be open and bounded in $Y_{0}$. Suppose that

$$
0 \notin H\left([0,1] \times{\overline{\partial_{Y_{0}} G}}^{\text {mod }}\right) \cap{\overline{H\left([0,1] \times \partial_{Y_{0}} G\right)}}^{\text {mod }} .
$$

By Lemma 3.9, there exists $X_{\lambda} \in \Lambda$ such that

$$
0 \notin H_{\mu}\left([0,1] \times \partial G_{\mu}\right) \text { for all } X_{\mu} \supset X_{\lambda} .
$$

Consequently,

$$
d_{\mu}\left(H_{\mu}(t, .), G_{\mu}, 0\right)=\text { constant for all } t \in[0,1] \text {, when } X_{\mu} \supset X_{\lambda} .
$$

Let $t_{1}, t_{2} \in[0,1]$ be arbitrary. Then

$$
d_{\mu}\left(H_{\mu}\left(t_{1}, .\right), G_{\mu}, 0\right)=d_{\mu}\left(H_{\mu}\left(t_{2}, .\right), G_{\mu}, 0\right),
$$


and going to the limit we obtain

$$
d\left(H\left(t_{1}, .\right), G, 0\right)=d\left(H\left(t_{2}, .\right), G, 0\right),
$$

which means that $d(H(t,), G, 0)=$. constant for all $t \in[0,1]$.

$\left(C_{4}\right)$ Let $K \in \mathcal{F}^{a}$ a mapping satisfying

$$
\langle K(u), u\rangle>0 \text {, when } u \neq 0 \text {, and } K(0)=0 \text {. }
$$

Suppose that $f \notin K\left({\overline{\partial_{Y_{0}}}}^{\text {mod }}\right) \cap{\overline{K\left(\partial_{Y_{0}} G\right)}}^{\text {mod }}$ and $f \in K(G)$. Let $u \in G$ be such that $K(u)=f$, and choose $X_{\lambda} \in \Lambda$ such that $u \in X_{\lambda}$. Then $\phi_{\mu}^{*}(f) \in K_{\mu}\left(G_{\mu}\right)$ for all $X_{\mu} \supset X_{\lambda}$. Moreover, $\left\langle K_{\mu}(v), v\right\rangle>0$ for every $v \in X_{\mu}, v \neq 0$. By the basic properties of the Brouwer degree, we have $d_{\mu}\left(K_{\mu}, G_{\mu}, \phi_{\mu}^{*}(f)\right)=1$ for all $X_{\mu} \supset X_{\lambda}$. Hence $d(K, G, f)=1$.

3.3. Properties of the degree function. Using the conditions $\left(C_{1}\right)-\left(C_{4}\right)$ for the degree function, we can deduce some standard properties.

Property 1. Let $F, T \in \mathcal{F}^{a}, G \subset Y_{0}$ open and bounded in $Y_{0}, F / \partial_{Y_{0}} G=T / \partial_{Y_{0}} G$ and $f \in Z_{0}$.

If $1<p^{-} \leq p(\cdot) \leq p^{+}<\infty$ and $f \notin F\left(\partial_{Y} G\right)$, then $d(F, G, f)=d(T, G, f)$.

Proof. Define an affine homotopy $H: D_{H} \rightarrow Z$,

$$
H(t, u)=t F(u)+(1-t) T(u),
$$

which belongs ti the class $\mathcal{H}$ by Lemma 3.4. It is clear that

$$
H\left([0,1] \times \partial_{Y_{0}} G\right)=F\left(\partial_{Y_{0}} G\right) .
$$

Since $f \notin F\left(\partial_{Y} G\right)$, we have $f \notin H\left([0,1] \times \partial_{Y} G\right)$. By homotopy invariance,

$$
d(F, G, f)=d(T, G, f) .
$$

Property 2. If $F \in \mathcal{F}$ and $G \subset Y_{0}$ is an open and bounded in $Y_{0}$.

If $1<p^{-} \leq p(\cdot) \leq p^{+}<\infty$, then $d(F, G,$.$) is constant on each open component in$ $Z_{0}$ of the open set $Z_{0} \backslash F\left(\partial_{Y} G\right)$.

Proof. Let $\Delta \subset Z_{0} \backslash F\left(\partial_{Y} G\right)$ be an open component in $Z_{0}$ and $f_{1}, f_{2} \in \Delta$ arbitrary. Then there exists a continuous curve $y:[0,1] \rightarrow Z_{0}$ such that $y(0)=f_{1}, y(1)=f_{2}$ and $y(t) \in \Delta$ for all $t \in[0,1]$. Therefore $y(t) \notin F\left(\partial_{Y} G\right)$. We see immediately that $F(u)-y(t) \in \mathcal{H}$ and $0 \notin F(\partial G)-y([0,1])$. By homotopy invariance, $d(F, G, y(0))=d(F, G, y(1))$ and we have the proof.

Property 3. Let $F \in \mathcal{F}, G \subset Y_{0}$ open and bounded in $Y_{0}$ and $u_{0} \in G$. Define a mapping $s: Y_{0} \rightarrow Y_{0}, s(u)=u-u_{0}$. If $0 \notin F\left({\overline{\partial_{Y_{0}} G}}^{\text {mod }}\right) \cap{\overline{F\left(\partial_{Y_{0}} G\right)}}^{\text {mod }}$, then

$$
d(F, G, 0)=d\left(F_{o s}^{-1}, s(G), 0\right) .
$$

Proof. Choose $X_{\lambda_{0}} \in \Lambda$ such that $u_{0} \in X_{\lambda_{0}}$. Now

$$
d(F, G, 0)=\lim _{\lambda \geq \lambda_{0}} d_{\lambda}\left(F_{\lambda}, G_{\lambda}, 0\right) .
$$

By the properties of the Brouwer degree, we have

$$
\left.d_{\lambda}\left(F_{\lambda}, G_{\lambda}, 0\right)=d_{\lambda}\left(F_{\lambda} o s^{-1}, s\left(G_{\lambda}\right), 0\right)=d_{\lambda}\left(\left(F_{o s}^{-1}\right)_{\lambda},(s(G))_{\lambda}\right), 0\right) .
$$


Moreover, it is easy to check that $F_{o s}{ }^{-1} \in \mathcal{F}, s(G) \subset Y_{0}$ is open and bounded in $Y_{0}$ and $0 \notin F\left({\overline{\partial_{Y_{0}} s(G)}}^{\text {mod }}\right) \cap{\overline{F\left(\partial_{Y_{0}} s(G)\right)}}^{\text {mod }}$. Therefore

$$
\left.d(F, G, 0)=\lim _{\lambda \geq \lambda_{0}} d_{\lambda}\left(\left(F_{o s}^{-1}\right)_{\lambda},(s(G))_{\lambda}\right), 0\right)=d\left(F_{o s}^{-1}, s(G), 0\right) .
$$

\section{REFERENCES}

[1] Berkovits,J.: On the degree theory for nonlinear mappings of monotone type. -Ann. Acad. Sci. Fenn. Ser. A I Math. Dissertationes 58,1986.

[2] Berkovits,J., and V.Mustonen: On topological degree for mappings of monotone type. Nonlinear Anal. 10,1986,1373-1383.

[3] Berkovits,J., and V.Mustonen: Nonlinear mappings of monotone type I. Classification and degree theory. Preprint No 2/88, Mathematics, University of Oulu.

[4] Brouwer, L.E. J: Uber Abbildung von Mannigfaltigkeiten. - Math. Ann. 71, 1912 ,97-115.

[5] F.E. Browder, Fixed point theory and nonlinear problems, Bull. Amer. Math. Soc. 9 (1983) 139.

[6] Browder,FE: Degree of mapping for nonlinear mappings of monotone type. Proc. Natl. Acad. Sci. USA 80, 1771-1773 (1983).

[7] Deimling,K: Nonlinar functional analysis. Springer, Berlin (1985).

[8] L. Dingien, P. Harjulehto, P. Hästö, M. Ruzicka: Lebesgue and Sobolev Spaces with Variable Exponents, Springer (2011).

[9] L. Fuhrer, Ein elementarer analytischer Beweis zur Eindeutigkeit des A bbildungsgrades im Rn, Math. Nachr. 54 (1972), 259-267.

[10] J.P. Gossez; Nonlinear elliptic boundary value problems for equations with rapidly (or slowly) increasing coefficients, Trans. Am. Math. Soc. 190 (1974), 163-205.

[11] O. Kováčik and J. Rákosník: On spaces $L^{p(x)}$ and $W^{1, p(x)}$, Czechoslovak Math. J. 41 (1991), 592-618.

[12] Leray, J, Schauder, J: Topologie et equationes fonctionnelles. Ann. Sci. Ec. Norm. Super. 51, 45-78 (1934).

[13] Landes, R., and V. Mustonen: Pseudo-monotne mappings in Orlicz-Sobolev spaces and nonlinear boundary value problems on unbounded domains. -J.Math. Anal. 88,1982,25-36.

[14] Narici, L., and E. Beckenstein: Topological vector spaces. -Marcel Dekker, Inc., New York and Basel, 1985.

[15] Skrypnik,IV: Nonlinear higher order elliptic equations. Naukova Dumka, Kiev (1973)(in Russian).

[16] Skrypnik,IV: Methods for analysis of nonlinear elliptic bondary value problems. Amer. Math. Soc. Transl., Ser. II, vol. 139. AMS, Providence(1994).

[17] H. Amann and S. Weiss, On the uniqueness of the topological degree, Math. Z. 130 (1973), 39-5.

[18] Zeidler, E: Nonlinear functional analysis and its applications I: Fixed-PointTheorems.Springer, New York (1985).

(M. Ait Hammou) Sidi Mohamed Ben Abdellah university, Department of MathematICS, P.C 1796, Fez, Morocco

Current address: Sidi Mohamed Ben Abdellah university, Department of Mathematics, P.C 1796, Fez, Morocco

E-mail address, M. Ait Hammou: mustapha.aithammou@usmba.ac.ma

(E. Azroul) Sidi Mohamed Ben Abdellah university, Department of Mathematics, P.C 1796, Fez, Morocco

E-mail address, E. Azroul: elhoussine.azroul@usmba.ac.ma 